\title{
Techniques for asynchronous and periodically synchronous coupling of atmosphere and ocean models
}

\section{Part II: impact of variability}

\author{
Reinhard Voss ${ }^{1}$, Robert Sausen ${ }^{2}$ \\ ${ }^{1}$ Deutsches Klimarechenzentrum, Bundesstr. 55, D-20146 Hamburg, Germany \\ ${ }^{2}$ Institut für Physik der Atmosphäre, DLR Oberpfaffenhofen, D-82234 Weßling, Germany
}

Received: 25 August 1995 / Accepted: 1 February 1996

\begin{abstract}
A periodically synchronous scheme suitable for coupling atmosphere and ocean models with high internal variability is presented. The performance of the scheme is tested by means of a simple zero-dimensional non-linear energy balance model with stochastic forcing. The equilibrium behaviour and the response to changes in the model parameters are analysed. The response experiments are similar to $\mathrm{CO}_{2}$ doubling and transient $\mathrm{CO}_{2}$ experiments. The best results are obtained using a method with weighted means of the airsea fluxes which are calculated during the synchronously coupled periods.
\end{abstract}

\section{Introduction}

Periodically synchronous and asynchronous coupling techniques enable a reduction of the computer time required by coupled atmosphere-ocean general circulation models. The reduction of the enormous amount of computational resources consumed by these models allows longer simulations or a higher spatial resolution of the models.

If a periodically synchronous coupling technique (Schlesinger 1979) is applied, periods with synchronous coupling (atmosphere and ocean are integrated quasisimultaneously) alternate with periods in which only the oceanic sub-model is integrated. As most of the computer time is usually consumed by the atmospheric part of a coupled model, this temporary switch-off of the atmospheric sub-model can reduce the computer time considerably, see Sausen and Voss (1996, hereafter cited as SV) for examples. On the other hand, this switch-off requires assumptions about the forcing required by the ocean during the ocean only periods. In the asynchronously coupled mode (e.g. Manabe and Bryan 1969) both sub-models are alternately inte-

Correspondence to: R. Voss grated. In this case the atmosphere only periods are much shorter than the ocean only periods in order to save a significant amount of computer time.

Asynchronous and periodically synchronous coupling techniques were analysed and/or applied by several groups (e.g. Schneider and Harvey 1986; Harvey 1986; Manabe and Stouffer 1988; Sausen 1988; Roberts 1990). In the first part of our study (SV) both techniques have been tested by means of a simple non-linear energy balance model. While the asynchronous coupling technique proved to be rather successful in the case of the model with annual mean forcing, this technique was not suitable for the annual cycle case. The periodically synchronous coupling method, on the other hand, could also be applied with good results to the model version with annual cycle forcing. However, both techniques fail as soon as the high internal variability of the atmosphere-ocean system is included.

Sausen (1988) made a suggestion on how to consider the high variability of the coupled model in the periodically synchronous coupling scheme. In this study we extend these ideas and examine the method by means of a simple non-linear energy balance model. In this model variability is introduced by an external stochastic forcing.

A description of the simple model is given in Sect. 2. Synchronously coupled reference experiments are presented in Sect. 3. In Sect. 4, a periodically synchronous coupling technique suitable for models including high variability is described. The quasi-equilibrium state and the transient behaviour of the periodically synchronously coupled model are investigated in particular in Sect. 5. In Sect. 6 the results are summarized.

\section{A simple atmosphere-ocean model}

We use a non-linear zero-dimensional atmosphereocean model in order to investigate the performance of different coupling schemes. In this section we only list the main features as a more detailed description of the model is given in SV. The model equations express the 
heat balance of the atmosphere in Eq. (1), the oceanic mixed layer in Eq. (2), and the deep ocean in Eq. (3), respectively (subscripts $A, O 1, O 2$ ).

$$
\begin{aligned}
& C_{A} \frac{d T_{A}}{d t}=R_{A}-\lambda_{\mathrm{A}} T_{A}+k_{1}\left(T_{O 1}-T_{A}\right) \\
& C_{O 1} \frac{d T_{O 1}}{d t}=R_{O}-\lambda_{\mathrm{O}} T_{O 1}-k_{1}\left(T_{O 1}-T_{A}\right)+k_{2}\left(T_{O 2}-T_{O 1}\right)
\end{aligned}
$$

$$
C_{O 2} \frac{d T_{O 2}}{d t}=-k_{2}\left(T_{O 2}-T_{O 1}\right)
$$

where $T_{A}, T_{O 1}$, and $T_{O 2}$ are the temperatures, $C_{A}, C_{O 1}$, and $C_{O 2}$ are the heat capacities, $\left(R_{A}-\lambda_{\mathrm{A}} T_{A}\right)$ and $\left(R_{O}-\lambda_{O} T_{O 1}\right)$ represent the radiative forcing, and $F=k_{1}\left(T_{O 1}-T_{A}\right)$ and $k_{2}\left(T_{O 2}-T_{O 1}\right)$ are, respectively, the fluxes of energy from ocean to atmosphere and from deep ocean to upper ocean. The standard values of the model constants are displayed in Table 1.

The non-linearity of the model is introduced by the formulation of the ice-albedo feedback which is implemented by the definition of $R_{O}$ (see also Fig. 1 of SV):

$R_{O}= \begin{cases}R_{O}^{(1)} & \text { for } T_{O 1} \leq T_{O}^{(1)} \\ R_{O}^{(1)}+\frac{T_{O 1}-T_{O}^{(1)}}{T_{O}^{(2)}-T_{O}^{(1)}}\left(R_{O}^{(2)}-R_{O}^{(1)}\right) & \text { for } T_{O}^{(1)}<T_{O 1}<T_{O}^{(2)} \\ R_{O}^{(2)} & \text { for } T_{O}^{(2)} \leq T_{O 1}\end{cases}$

The temperature-independent part of the radiative forcing of the atmosphere, $R_{A}$, is given by

$R_{A}=R_{A}^{(1)}+R_{A}^{(2)} \sin (\omega t)+R_{A}^{(3)} \xi(t)$.

In addition to the annual mean forcing $R_{A}^{(1)}$ and the annual cycle forcing $R_{A}^{(2)} \sin (\omega t)$, which were already used by SV, a stochastic forcing $R_{A}^{(3)} \xi(t)$ is introduced. Here $\xi(t)$ represents white noise with variance 1.0. Without the stochastic forcing the energy balance model would not produce variability. By means of this stochastic term, which was suggested by Sausen (1988), a characteristic feature of more comprehensive models and of the real climate system is introduced into the model: variability with a variance that can be regulated by the factor $R_{A}^{(3)}$.
Model runs performed with stochastic forcing should by analogy to integrations of coupled atmosphere-ocean general circulation models result in a totally different time evolution of the model for slightly different initial conditions. On the other hand, $\xi(t)$ should be reproducible in order to be able to repeat experiments. These requirements are accomplished by producing a series of randomly distributed numbers. The numbers are normally distributed (Gaussian frequency distribution) with a mean of 0.0 and a variance of 1.0. In order to simulate different initial conditions certain numbers at the beginning of the series are skipped. Every atmospheric time step, the third digit after the decimal point of the atmospheric temperature $T_{A}$ determines how many numbers of the series of random numbers are skipped next. This procedure assures that the random forcing is different from one run to another, either due to different initial conditions or due to differences in the coupling scheme.

\section{Synchronously coupled integrations}

Before we analyse the performance of the periodically synchronous coupling schemes, it is necessary to investigate our simple model in the synchronous coupled mode. These experiments serve as reference for the tests performed in the periodically synchronously coupled mode.

First, we consider control integrations performed with the standard value of the atmospheric emissivity $\left(\lambda_{A}=0.5245 \mathrm{Wm}^{-2} \mathrm{~K}^{-1}\right)$ and different amplitudes of the stochastic forcing $\left(R_{A}^{(3)}=0.0,32.5,65.0,130.0\right.$ $\mathrm{Wm}^{-2}$ ). Each experiment starts after a sufficient long spin-up period from the respective quasi-equilibrium state. As the external stochastic forcing leads to a rather high model variability, the experiments are integrated over 10000 years. The long-term averages of the annual mean temperatures of the atmosphere, the oceanic mixed layer, and the deep ocean exhibit a rise with increasing amplitude of the stochastic forcing (Table 2). Due to the model formulation (cf. Eq. 1-Eq. 3) the quasi-equilibrium temperatures of upper and deep ocean are identical.
Table 1. Standard values of the model constants

\begin{tabular}{lll}
\hline & Atmosphere & Ocean \\
\hline Heat capacity & $C_{A}=10^{7} \mathrm{Jm}^{-2} \mathrm{~K}^{-1}$ & $\begin{array}{l}C_{O 1}=10^{8} \mathrm{Jm}^{-2} \mathrm{~K}^{-1} \\
C_{O 2}=10^{9} \mathrm{Jm}^{-2} \mathrm{~K}^{-1}\end{array}$ \\
Solar input & $R_{A}^{(1)}=130.0 \mathrm{Wm}^{-2}$ & $R_{O}^{(1)}=120.0 \mathrm{Wm}^{-2}$ \\
& $R_{A}^{(2)}=65.0 \mathrm{Wm}^{-2}$ & $R_{O}^{(2)}=125.0 \mathrm{Wm}^{-2}$ \\
Reference & $R_{A}^{(3)}=65.0 \mathrm{Wm}^{-2}$ & \\
temperature & & $T_{O}^{(1)}=290.0 \mathrm{~K}$ \\
Emissivity & $T_{O}^{(2)}=295.0 \mathrm{~K}$ \\
Coupling constant & $k_{1}=0.5245 \mathrm{Wm}^{-2} \mathrm{~K}^{-1}$ & $\lambda_{O}=0.3472 \mathrm{Wm}^{-2} \mathrm{~K}^{-1}$ \\
Time step & $\Delta t_{A}=0.5 \mathrm{~d}$ & $k_{2}=0.2 \mathrm{Wm}^{-2} \mathrm{~K}^{-1}$ \\
\hline
\end{tabular}


Table 2. Averaged annual mean temperatures and the corresponding standard deviations of the quasi-equilibrium state calculated from 10000 year model output simulated by the synchronously coupled model with different amplitudes of the stochastic forcing $R_{A}^{(3)}$

\begin{tabular}{|c|c|c|c|c|c|c|}
\hline \multirow{2}{*}{$\begin{array}{l}\text { Stochastic } \\
\text { forcing } \\
R_{A}^{(3)} \\
{\left[\mathrm{Wm}^{-2}\right]}\end{array}$} & \multicolumn{3}{|c|}{ Annual mean temperature $[\mathrm{K}]$} & \multicolumn{3}{|c|}{ Standard deviation $[\mathrm{K}]$} \\
\hline & $\begin{array}{l}\text { Atmos- } \\
\text { phere }\end{array}$ & $\begin{array}{l}\text { Oceanic } \\
\text { mixed } \\
\text { layer }\end{array}$ & $\begin{array}{l}\text { Deep } \\
\text { ocean }\end{array}$ & $\begin{array}{l}\text { Atmos- } \\
\text { phere }\end{array}$ & $\begin{array}{l}\text { Oceanic } \\
\text { mixed } \\
\text { layer }\end{array}$ & $\begin{array}{l}\text { Deep } \\
\text { ocean }\end{array}$ \\
\hline 0.0 & 286.2 & 288.2 & 288.2 & 0.0 & 0.0 & 0.0 \\
\hline 32.5 & 286.2 & 288.2 & 288.2 & 0.5 & 0.5 & 0.1 \\
\hline 65.0 & 286.3 & 288.3 & 288.3 & 1.0 & 1.0 & 0.2 \\
\hline 130.0 & 286.7 & 288.8 & 288.8 & 2.2 & 2.1 & 0.5 \\
\hline
\end{tabular}

In Table 2, the variability is described by the standard deviation of the annual mean temperature. As expected, the standard deviations increase with increasing stochastic forcing factor, $R_{A}^{(3)}$. The values for the atmosphere and the oceanic mixed layer are of rather similar magnitude due to the strong coupling of both sub-systems. The deep ocean with its much longer relaxation time and its weaker coupling to the upper ocean (cf. SV) exhibits a much lower variability.

If the experiments with different $R_{A}^{(3)}$ were performed with the linear version of the model (i.e. setting $R_{O}^{(1)}=R_{O}^{(2)}$ in Eq. 4) the mean states of all experiments would be identical, and only the standard deviations would increase with increasing $R_{A}^{(3)}$. Therefore, it can be concluded that the differences of the quasi-equilibrium states are caused by the non-linear ice-albedo feedback. Apart from this described interannual variability, the model also exhibits intra-annual variability. Together with the annual cycle, the latter can lead to oceanic mixed layer temperatures temporarily higher than $T_{O}^{(1)}=290 \mathrm{~K}$ which marks the transition from a totally to partly ice-covered ocean (Eq. 4). The chance of exceeding $T_{O}^{(1)}$ depends on the amplitude of the annual cycle $\left(R_{A}^{(2)}\right)$ and the stochastic portions $\left(R_{A}^{(3)}\right)$ of the forcing. With increasing amplitude of the stochastic forcing, the chance for exceeding $T_{O}^{(1)}$ increases. Due to a positive feedback, the result is an increase of the mean temperature.

In the following, only experiments with $R_{A}^{(3)}=65.0$ $\mathrm{Wm}^{-2}$ are presented. As can be seen in Table 2, the standard deviations of the annual mean temperature are $1.0 \mathrm{~K}$ for the atmosphere and the oceanic mixed layer and $0.2 \mathrm{~K}$ for the deep ocean for this value of $R_{A}^{(3)}$.

Figure 1 shows the mean annual cycles of the temperatures $T_{A}, T_{O 1}, T_{O 2}$, and of the heat flux $F$ from the ocean to the atmosphere based on a 200 year integration of the model in its quasi-equilibrium state for $R_{A}^{(3)}=65.0 \mathrm{Wm}^{-2}$ and the standard atmospheric emissivity $\lambda_{A}$. In addition the $95 \%$ significance thresholds (i.e. 1.96 standard deviations) are plotted (dashed curves). If the temperatures of different years were normally distributed, $95 \%$ of the values would be found between these thresholds. Since in this plot only a 200 year period is considered the annual mean values (thin lines) are not identical to the values of the 10000 year integration displayed in Table 2. The phase displacement of approximately 2 months between the annual cycles of $T_{A}$ and $T_{O 1}$ is due to the slower adjustment of the oceanic mixed layer. Therefore, the resulting heat flux $F$ from the ocean to the atmosphere which is determined by the differences between $T_{O 1}$
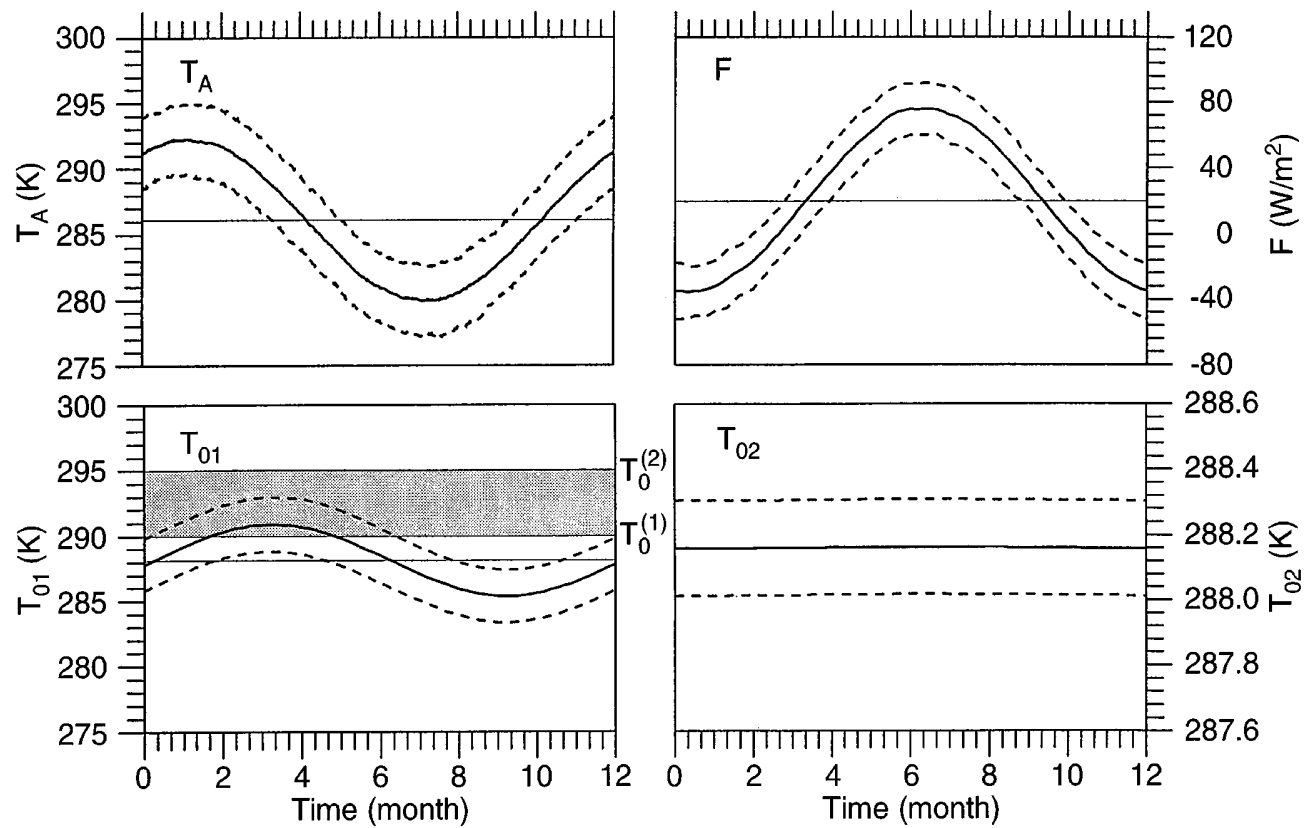

Fig. 1. Mean annual cycles (solid thick curves) of a 200 year control integration with the synchronously coupled model for the quasi-equilibrium state corresponding to the standard atmospheric emissivity $\lambda_{A}$. The dashed curves display the $95 \%$ significance thresholds (i.e. 1.96 standard deviations). The thin lines mark the annual means. The shaded area indicates the transition range between totally ice-covered and ice-free 
and $T_{A}$, exhibits its maximum just before the atmosphere reaches its temperature minimum. On an average, $T_{O 1}$ is larger than $T_{O}^{(1)}$ approximately 2 months of a year, and the sea surface is only partly ice-covered. For the rest of the year, the ocean is totally ice-covered. Due to variability, extreme years with the sea surface totally ice-covered throughout the annual cycle or with periods with partial ice-coverage two to three time longer can also occur. The deep ocean exhibits no visible annual cycle due to its long time constant and its weak coupling to the upper ocean.

As in SV, we tested the performance of periodically synchronous coupling schemes by comparing the results of the perturbation experiments with corresponding synchronously coupled experiments. Two different reference experiments with a $2 \%$ reduction of the atmospheric emissivity (from $\lambda_{A}$ to $\lambda_{A(\text { red })}$ ) were performed. We chose both an instantaneous reduction at $t=0$ and a gradual reduction during the first $100 \mathrm{simu}$ lated years (cf. Fig. 4 of SV). One cannot expect two corresponding synchronously and periodically synchronously coupled runs to match any better than two
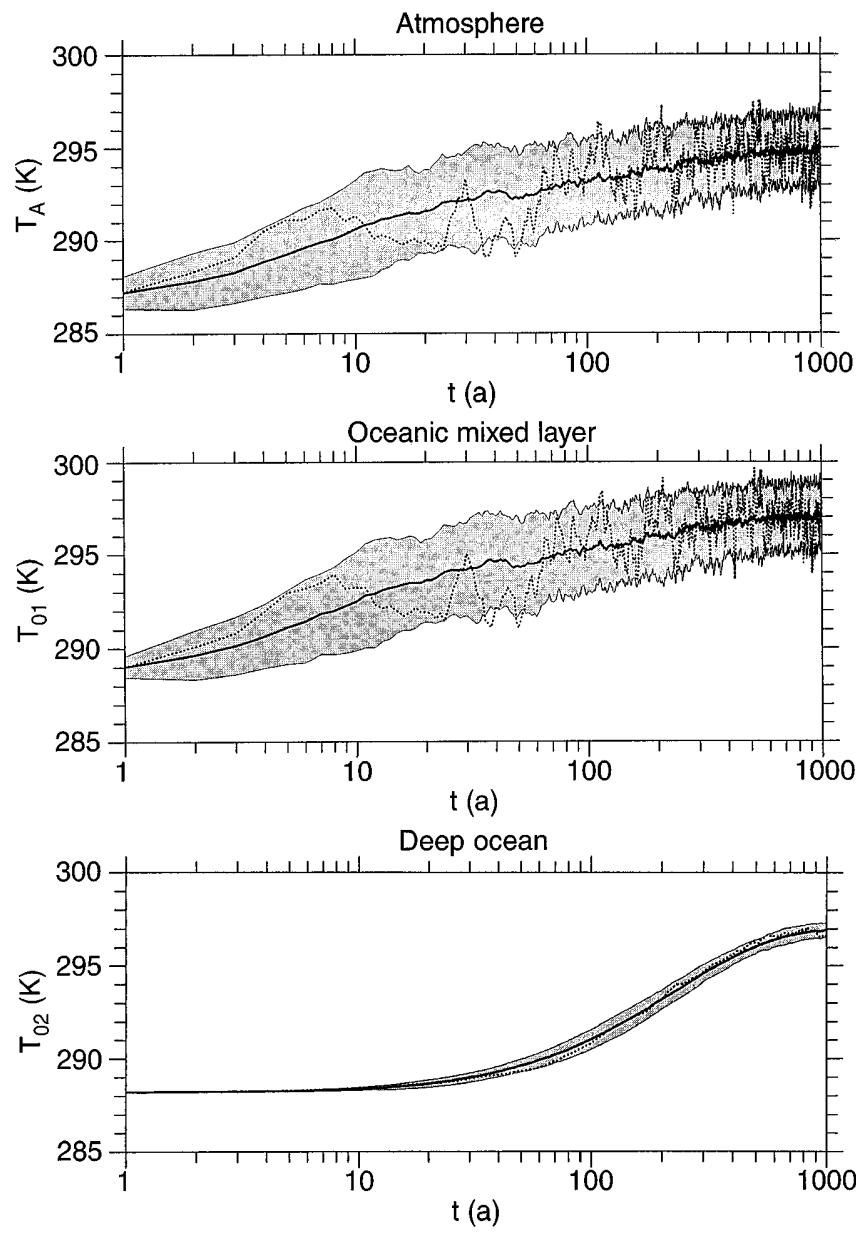

Fig. 2. The annual mean temperature response of a synchronously coupled experiment to the instantaneously reduced atmospheric emissivity at $t=0$. Displayed are the ensemble means of $50 \mathrm{re}-$ alisations (solid curves) and a single realisation (dotted curves). The range in which $95 \%$ of the annual means of the individual realisations occur is shaded different synchronously coupled runs would. Thus, we compare ensembles of synchronously and periodically synchronously coupled runs, respectively. Each ensemble consists of 50 simulations with different initialisations of the random forcing. All individual integrations start from the same initial state obtained from the model with the standard atmospheric emissivity $\lambda_{A}$ in the quasi-equilibrium state.

As an example, Fig. 2 shows the response of the synchronously coupled model to the instantaneous reduction of the atmospheric emissivity. The ensemble means of the annual mean temperatures for all three boxes (atmosphere, oceanic mixed layer, deep ocean) are shown. In order to show both the initial phase and the phase of asymptotic approach to the new quasiequilibrium, a logarithmic time axis is used. The shaded areas mark the range in which $95 \%$ of the annual mean temperatures of the individual runs occur when a normal distribution is assumed. In all three sub-systems, the ensemble means of the temperature (solid curves) increases approximately $9.0 \mathrm{~K}$ during the first 1000 simulated years. Since all integrations start from the same initial point the range in which the individual model trajectories occur is reduced in the first years. This feature could be eliminated, if each integration started from a different point of the model trajectory in the quasi-equilibrium state. For convenience we preferred to start the model runs from identical initial conditions as this has minor impact on the results. In contrast to the oceanic mixed layer and the atmosphere, the response of the deep ocean is delayed and the variability in this sub-system is much weaker. One realisation arbitrarily selected from the 50 integrations is additionally plotted (dotted curves). This single realisation illustrates the superposition of strong fluctuations and the adjustment process to the reduced atmospheric emissivity $\lambda_{A(\text { red })}$.

\section{A suitable periodically synchronous coupling scheme}

Periodically synchronous coupling methods consist of alternately synchronous and ocean only integration periods (cf. SV). In the synchronous mode both models are integrated quasi-simultaneously over the same time period, whereas in the other mode only the oceanic sub-model is integrated. During the latter periods, the ocean is forced by air-sea fluxes determined from the fluxes calculated during the previously integrated synchronous periods.

The efficiency of the periodically synchronously coupled model strongly depends on the length of the ocean only periods and on the fraction of computer time consumed by the atmospheric component in the corresponding synchronously coupled model. Figure 3 displays the consumed computer time of the periodically synchronously coupled model as a function of the ratio between the period lengths of the ocean only and synchronously coupled periods $\tau_{o c e} / \tau_{\text {syn }}$. Five examples are shown where the fraction of the atmospheric com- 


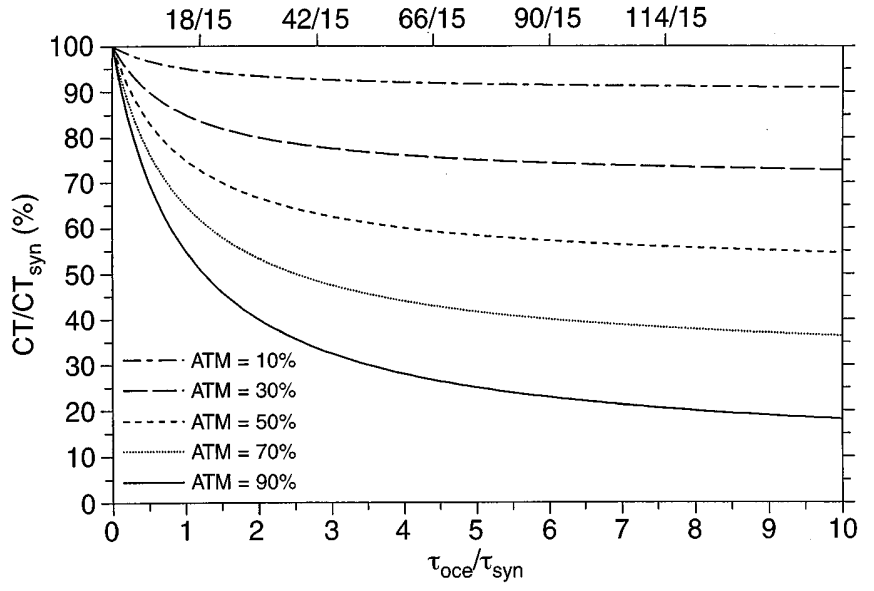

Fig. 3. The consumed computer time of periodically synchronously coupled models $(C T)$ as percent of the corresponding computer time of the synchronously coupled model $\left(C T_{s y n}\right)$ and as a function of the ratio between the length of the ocean only and synchronously coupled periods $\tau_{\text {oce }} / \tau_{\text {syn }}$. Examples are displayed for different fractions of computer time consumed by the atmospheric component of the synchronously coupled model. The ratios at the top of the figure indicate values of $\tau_{o c e} / \tau_{s y n}$ frequently used in the study

ponent varies between $10 \%$ and $90 \%$. The saving of computer time increases with increasing fraction of the atmospheric component and with increasing ratio $\tau_{\text {oce }}$ $\tau_{\text {syn }}$. In all cases a change of $\tau_{\text {oce }} / \tau_{\text {syn }}$ has the strongest effect for small values of the ratio. With increasing ratio of the period length the consumed computer time converges to the fraction of computer time required by the oceanic model.

If no stochastic forcing is included $\left(R_{A}^{(3)}=0\right)$, the best results are obtained using a method including an extrapolation of the fluxes during the ocean only periods (see SV). In a model without high temporal variability only two or three values of the fluxes during the synchronous periods are sufficient in order to determine the extrapolation coefficients. However, this method fails if the coupled model, especially the atmospheric sub-model, produces high variability. Due to the variability, single extreme events can affect the determination of the fluxes for the ocean only periods. By chance, the atmospheric temperature may be, for instance, relatively low at the time when the air-sea flux is determined. Consequently, the flux would be relatively large for the next ocean only period and the oceanic mixed layer would cool considerably. Apart from a rather strong deviation from the "true" response as simulated by the synchronously coupled model, this procedure may finally result in numerical instability, in particular, if an extrapolation is applied. Such problems can be avoided if a larger number of previously calculated synchronous periods is considered. Then more values of the fluxes are available in order to capture the long-term trend of the coupling flux. Sausen (1988) suggested a practicable way out of this problem and, in principle, demonstrated its power to solve the problem. However, rigorous tests were not

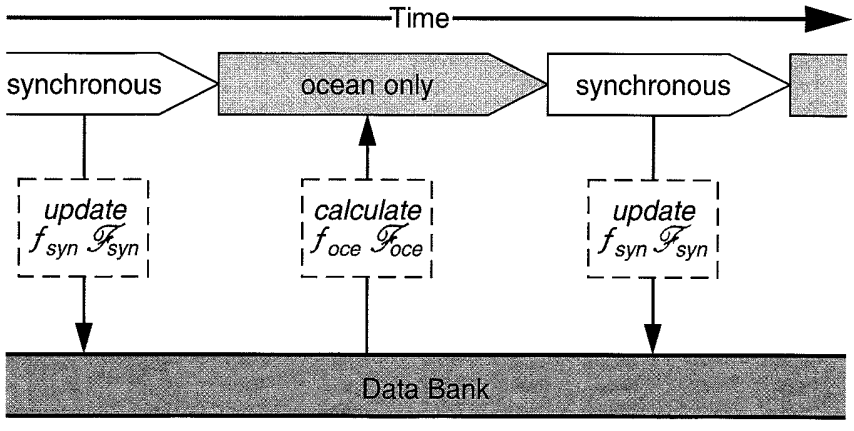

Fig. 4. Scheme of the periodically synchronous coupling method

made. In our investigation, we extend the idea of periodically synchronous coupling with weighted fluxes.

During the last 12 months of each synchronous period the values $f_{\text {syn }}(\hat{t})$ of the ocean forcing are stored in a data bank (Fig. 4). The ocean forcing consists of the 5 -day means of the heat flux $F$ calculated by the atmospheric sub-model for each ocean time step. In addition to the individual values $f_{\text {syn }}(\hat{t})$, the corresponding annual mean heat flux $\mathscr{F}_{\text {syn }}$ is also stored in the data bank. Here $\hat{t}$ denotes the day in the course of the year. At the end of each synchronous period the forcing for the following ocean only integration is calculated. The annual mean of the following ocean only integration $\mathscr{F}_{\text {cce }}$ results from the weighted annual mean of the last ocean only period $\mathscr{F}_{\text {oce(old })}$ and the annual mean of the latest 12 months of the newly completed synchronous period $\mathscr{F}_{\text {syn }}$ :

$\mathscr{F}_{\text {oce }}=\frac{g \mathscr{F}_{\text {oce }(\text { old })}+\mathscr{F}_{\text {syn }}}{g+1}$

where the weight $g \geq 0$. The values $f_{\text {oce }}(\hat{t})$, which are taken as forcing in the course of the year during the following ocean only period, are determined according to

$f_{\text {oce }}(\hat{t})=f_{\text {syn }}(\hat{t})-\mathscr{F}_{\text {syn }}+\mathscr{F}_{\text {oce }}$,

i.e. the annual mean of the flux $\mathscr{F}_{\text {syn }}$ is replaced by the weighted mean $\mathscr{F}_{\text {oce }}$. As stated $f_{\text {syn }}(\hat{t})$ are the individual values of the heat flux as simulated during the last 12 months of the just finished synchronously coupled period. The data set $f_{\text {oce }}(\hat{t})$, which comprises annual mean plus annual cycle, is used as forcing in each year of following ocean only period.

The separation of the annual mean fluxes from the intra-annual fluctuations saves a part of the high variability of the model. At the same time, the long-term trend is prevented from unrealistic excursions to large positive or negative values during the ocean only periods where a feedback between the oceanic state and the heat flux $F$ is not allowed. Sausen (1988) also applied a weighting procedure to the annual cycle. However, we found by means of rigorous tests that a better reproducibility of the synchronously coupled simulations is obtained when the annual cycle including the short time variability is not damped. This high frequen- 
cy can affect the long-term fluctuations of the coupled system and should therefore be retained (e.g. Hasselmann 1988).

Let $\mathscr{F}_{\text {oce }}^{(n)}$ and $\mathscr{F}_{\text {syn }}^{(n)}$ denote the annual mean heat fluxes during the $\mathrm{n}$-th ocean only period and $\mathrm{n}$-th synchronous period, respectively. Then the recursion formula Eq. (6) can be transformed into an expansion series. When $g=0$ the result is

$\mathscr{F}_{\text {oce }}^{(n)}=\mathscr{F}_{\text {syn }}^{(n)}$.

When $g>0$ we obtain

$\mathscr{F}_{\text {oce }}^{(1)}=\mathscr{F}_{\text {syn }}^{(1)}$,

$\mathscr{F}_{\text {oce }}^{(n)}=\left(\frac{g}{g+1}\right)^{n+1} \mathscr{F}_{\text {syn }}^{(1)}+\frac{1}{g+1} \sum_{i=0}^{n-2}\left(\frac{g}{g+1}\right)^{i} \mathscr{F}_{\text {syn }}^{(n-i)}$

for $n \geq 2$. Letting

$G_{i}=\frac{1}{g+1}\left(\frac{g}{g+1}\right)^{i}$

for $i=0,1,2, \ldots$ Equation (10) can be transformed into

$\mathscr{F}_{\text {oce }}^{(n)}=g G_{n-2} \mathscr{F}_{\text {syn }}^{(1)}+\sum_{i=0}^{n-2} G_{i} \mathscr{F}_{\text {syn }}^{(n-i)}$

for $n \geq 2$. For $g>0$ the weighting factors $G_{i}$ decrease with increasing $i$, i.e. with increasing time distance to the newly finished synchronous period. In Fig. 5, the weighting factors $G_{i}$ of the individual annual means $\mathscr{F}_{s y n}^{(n-i)}$ of Eq. (12) are shown for different weights $g$. In all cases, the annual mean of the latest synchronous pe$\operatorname{riod} \mathscr{F}_{s y n}^{(n)}$ has the largest weighting factor but its magnitude relative to the weighting factors of older annual means depends heavily on $g$. With increasing $g$ the importance of the latest annual mean decreases (i.e. $G_{0}$ decreases) and the information of previously calculated values becomes more important for the forcing of the following ocean only period. Consequently, the effect of a single annual mean $\mathscr{F}_{s y n}^{(n-i)}$ on $\mathscr{F}_{\text {oce }}^{(n)}$ also decreases.

When our periodically synchronous coupling scheme is applied the recursion formula Eq. (6) is used. Thus, only $\mathscr{F}_{o c e}^{(n)}, \mathscr{F}_{\text {syn }}^{(n)}$ and $f_{\text {syn }}(\hat{t})$ are stored in the data bank. Before the periodically synchronous experiments are started, the data bank is filled with heat fluxes of the previously synchronously coupled integration in the quasi-equilibrium state for the standard atmospheric emissivity $\lambda_{A}$.

\section{The performance of the periodically synchronous coupling technique}

\subsection{Simulations performed with the standard value of atmospheric emissivity}

First, we analyse to what extent the quasi-equilibrium state corresponding to the standard atmospheric emissivity $\lambda_{A}$ is reproduced by the periodically synchronous

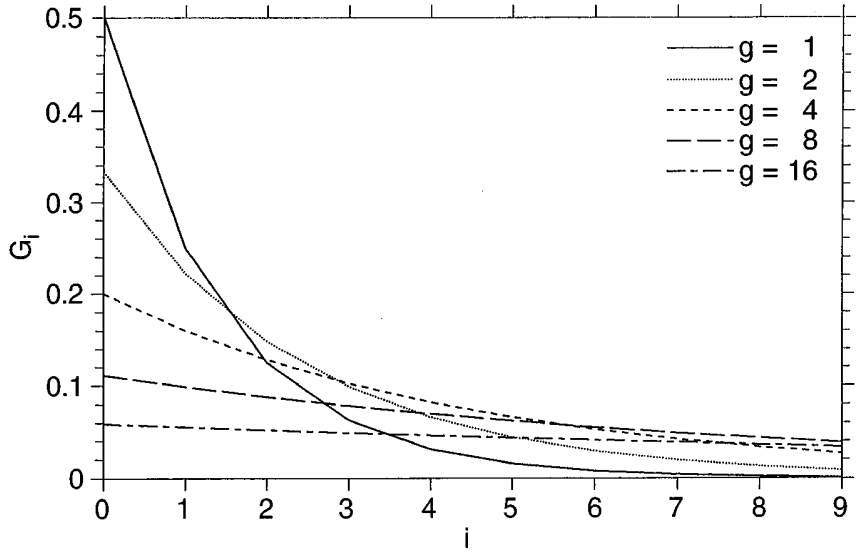

Fig. 5. Weighting factors $G_{i}$ of the individual annual mean fluxes $\mathscr{F}_{s y n}^{(n-i)}$ of the synchronous periods for different weights $g$

method for various combinations of the weight $g$ and the length of the ocean only periods $\tau_{\text {oce }}$. The weight $g$ takes the values $0,1,2, \ldots, 16$. The length of the ocean only periods takes the values $18,42,66,90$ and 114 months. In all cases the period of the synchronous coupling $\tau_{s y n}$ lasts 15 months. The corresponding ratios $\tau_{o c e} / \tau_{o c e}$ are indicated in Fig. 3. The first three months of each synchronous period are not stored in the data bank. This period is characterised by the spin-up process of the atmosphere to the new ocean state reached in the newly finished ocean only period. Thus, an impact of the spin-up on the forcing for the next ocean only period is avoided. The simulation period of the individual experiments is 15000 years. In order to avoid an, in principle possible, climate drift affecting the results, the annual means and the corresponding standard deviations of only the last 10000 years are considered. Even in view of the rather long time constant of the deep ocean (160 years, see SV) 5000 years appears to be a sufficient adjustment time. The results are compared to the respective values obtained from the synchronously coupled control simulation (see Table 2).

First we consider the cases without weighting the annual mean heat flux $(g=0)$. This procedure is identical to the method without extrapolation applied by SV. The error in the annual mean equilibrium temperature of the oceanic mixed layer increases with increasing $\tau_{\text {oce }}$ (Fig. 6a). The reproducibility of the equilibrium temperatures is improved for $g>0$. Beyond $g=3$ no significant dependence of the errors on $g$ or $\tau_{\text {oce }}$ can be observed. The corresponding temperature errors of the deep ocean and the atmosphere are very similar since the model formulation demands the same equilibrium temperatures for both ocean boxes and due to the strong atmosphere-ocean coupling. Compared to the corresponding standard deviations of the synchronously coupled control run $(1.0 \mathrm{~K}$ for the oceanic mixed layer and $0.2 \mathrm{~K}$ for the deep ocean, see Table 2), the errors of the periodically synchronously coupled experiments, particularly the experiments performed with $g>0$, remain in the range of fluctuations of the synchronously coupled experiment. 


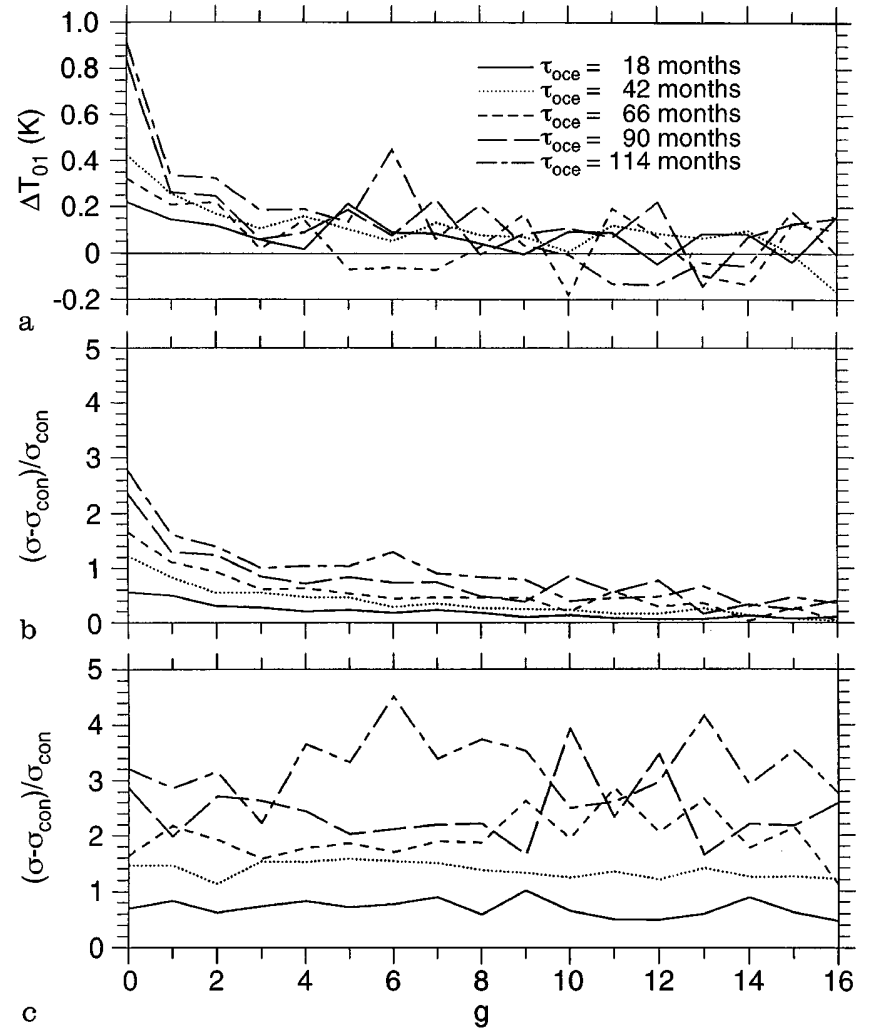

Fig. 6. a Differences of the quasi-equilibrium oceanic mixed layer temperatures between periodically synchronously coupled experiments and the corresponding synchronously coupled control experiment. The corresponding standard deviations $\sigma$ of $\mathbf{b}$ the oceanic mixed layer and $\mathbf{c}$ deep ocean temperature are calculated as relative deviations from the values of the synchronously coupled control experiment $\sigma_{c o n}$

Due to its non-linearity, the equilibrium state of the simple model depends on the internal variability (cf. Sect. 3). A bias in the model variability leads to an incorrect equilibrium state of the model. The standard deviation $\sigma$ of the annual mean temperatures is used as a measure for the variability. In Fig. $6 b$, c, the relative deviations from the corresponding values of the synchronously coupled control experiment $\sigma_{c o n}$ is displayed for the oceanic mixed layer and the deep ocean. Common to all periodically synchronously coupled experiments is a larger standard deviation than in the reference case, particularly in the deep ocean. In other words, the periodically synchronously coupled model can reach states that do not occur in the corresponding synchronously coupled run. These stronger excursions of the temperature are due to the missing feedback between the oceanic mixed layer temperature and the air-sea flux during the ocean only periods. Both Fig. $6 \mathrm{~b}, \mathrm{c}$, show the general trend that a shortening of $\tau_{\text {oce }}$ improves the reproducibility of the variability. In accordance with the errors in the equilibrium temperatures, the worst results in the oceanic mixed layer are achieved without weighting $(g=0)$. Whereas the standard deviation in the oceanic mixed layer decreases with increasing $g$, the impact of $g$ on the variability of the deep ocean is negligible.

\subsection{The response to an instantaneous reduction of the atmospheric emissivity}

One important application of the periodically synchronous coupling method is its use for coupled atmosphere-ocean model simulations to assess climate change. As mentioned in Sect. 3, we test the periodically synchronous coupling technique in an experiment where the atmospheric emissivity $\lambda_{A}$ is instantaneously reduced by $2 \%$ to $\lambda_{A(\text { red })}$ at $t=0$ (see Fig. 4 of SV). The length of the ocean only periods $\tau_{\text {oce }}$ increases from rather short values at the beginning of the simulation until a final value is reached. This technique was proposed by Harvey (1986) and has been successfully applied to the simple model without stochastic forcing (SV). The first synchronous period lasts 24 months and all following synchronous periods last 15 months. Only the last 12 months of each synchronous period are stored in the data bank (see Sect. 4). The ocean only integration periods $\tau_{\text {oce }}$ last $6,12,18,24,30,39,48,57$ months and for the rest of the simulation 66 months are used.

In Fig. 7, the ensemble means of 50 realisations of the periodically synchronously coupled model with weights $g$ of $0,2,4$ and 8 are compared to the corresponding results of the synchronously coupled model shown in Fig. 2. The shaded areas indicate the range in which $95 \%$ of the annual means of the synchronously coupled experiment occur if a normal distribution is assumed. During a period of 5 years after the first synchronous period, which lasts 24 months, the oceanic mixed layer temperature $T_{O 1}$ is slightly underestimated in all experiments. The forcing of the ocean only periods is determined from the heat fluxes of the newly finished synchronously coupled periods and for $g>0$ additionally from older synchronous periods. Because of the rapid changes (in particular of the heat flux) during the first simulated years this determination of the forcing causes a delayed response.

After approximately 10 simulated years the errors of $T_{O 1}$ depend on the value of $g$. Using fluxes of the newly finished synchronous period $(g=0)$ leads to an underestimation of the temperature whereas with increasing $g$ this underestimation changes to an overestimation with errors up to $2.0 \mathrm{~K}$ for the experiment with $g=8$. A comparison between these errors and the time evolution of the synchronously coupled experiment (Fig. 2) shows that the large errors are connected with an overshooting of the temperature. This overshooting happens at a period when the strong rapid model response changes to a weaker slower one. Due to the time delay, which is inherently present in the periodically synchronous coupling technique (especially for $g>0$ ), the reduction in the change rate is delayed.

The deep ocean exhibits a qualitatively similar behaviour. Relative to the variability of the synchronous reference run (Fig. 2) the errors are larger. However, 

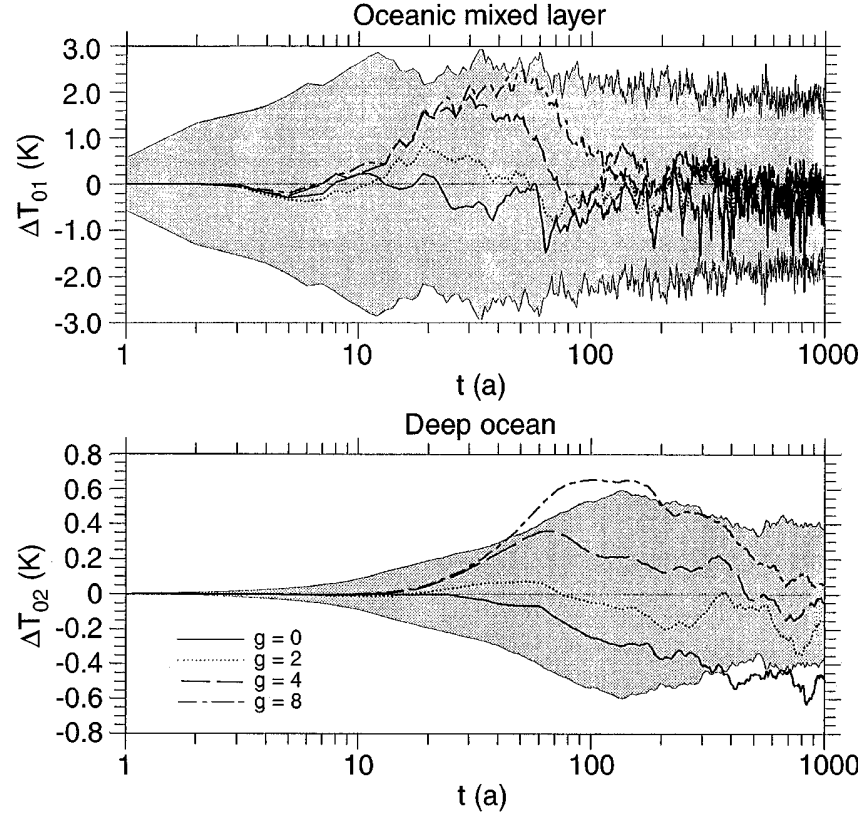

Fig. 7. Errors of the ensemble mean responses to the instantaneously reduced atmospheric emissivity as simulated by the periodically synchronously coupled model with different weights $g$. Shaded areas indicate the range in which $95 \%$ of the values of the synchronously coupled reference experiment occur

the maximum values of approximately $0.6 \mathrm{~K}$ are much smaller than in the upper ocean.

The evolution of the deep ocean temperature errors indicates that the periodically synchronously coupled model does not inevitably reach the same equilibrium state as the reference experiment. As with the results of Sect. 5.1, single integrations over 15000 years show slight deviations in the new quasi-equilibrium state. As the new oceanic mixed layer temperature is above the threshold $T_{O}^{(2)}$ (cf. Sect. 3), i.e. the ocean is mostly icefree, the equilibrium temperature of the periodically synchronously coupled runs tend to be underestimated for the same reasons causing the overestimation in Sect. 5.1 .

In order to examine the transient behaviour of the coupled model in more detail, a series of experiments with weights $g$ ranging from 0 to 16 with increments of 1 and increasing ocean only periods $\tau_{\text {oce }}$ with a final length between 18 and 114 months were performed. Each experiment consists of 50 realisations. The ensemble means are compared to the corresponding synchronously coupled reference experiment. As shown in Fig. 7, the largest temperature errors occur during the first 200 simulated years. Hence, the root mean square (rms) errors of the oceanic mixed layer temperature for this period are shown in Fig. 8. For experiments performed with the same weight, the errors increase with increasing length of the ocean only periods $\tau_{\text {oce }}$. The longer $\tau_{\text {oce }}$ the cheaper the simulation is. In other words, the errors increase with decreasing consumption of computer time. The smallest rms errors, which occur for $g=1$ or $g=2$ depending on $\tau_{\text {oce }}$, are associated with the change from an underestima-

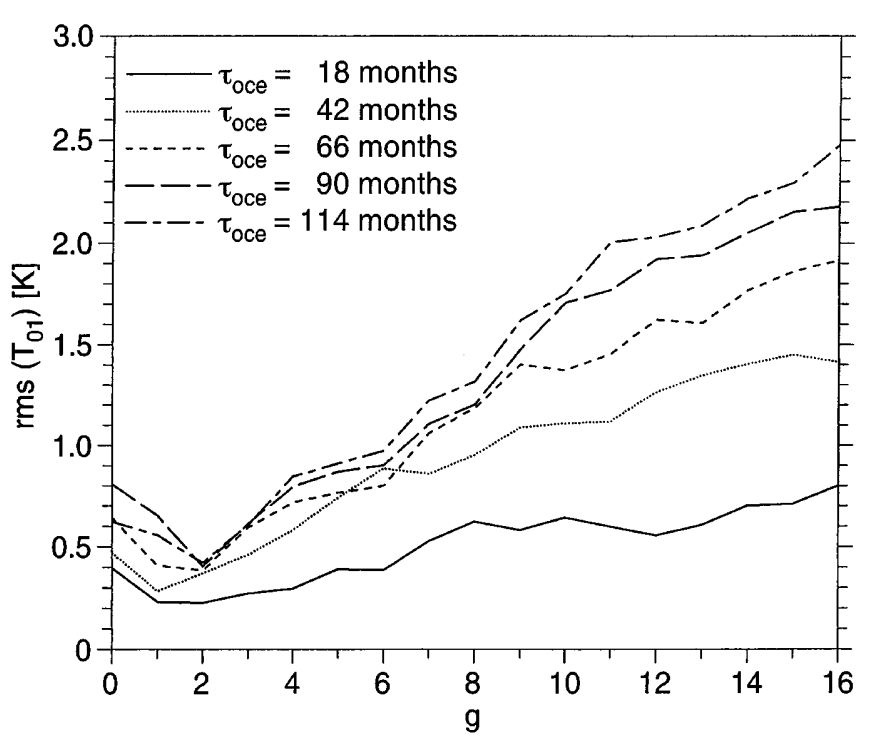

Fig. 8. The ensemble means of root mean square errors of the annual mean oceanic mixed layer temperature of the first 200 simulated years. The experiments are performed with instantaneously reduced atmospheric emissivity for different weights $g$ and different lengths of the ocean only integration periods $\tau_{\text {oce }}$

tion to an overestimation of $T_{O 1}$ (see Fig. 7). Forcing the ocean during the ocean only periods with unchanged fluxes (i.e. $g=0$ ) or too strongly damped fluxes of the newly finished synchronously coupled period, leads to unfavourably large errors in the first 200 simulated years of the experiments. The behaviour of the deep ocean temperature is similar (not shown).

\subsection{The response to a gradual reduction of the atmospheric emissivity}

In order to analyse a more realistic climate change scenario we chose a gradual reduction of the atmospheric emissivity during the first 100 years after which the atmospheric emissivity is kept constant at the value $\lambda_{A(\text { red })}$ (cf. Fig. 4 of SV). This is similar to a transient $\mathrm{CO}_{2}$ increase whereas the previously used instantaneous reduction of the atmospheric emissivity (Sect. 5.2) is similar to a traditional $\mathrm{CO}_{2}$ doubling experiment. Both scenarios start with the same initial value $\lambda_{A}$ and reach the same final value $\lambda_{A(r e d)}$. Therefore, experiments performed with both types of emissivity reduction should approach the same quasi-equilibrium state as our model does not have multiple equilibria.

Figure 9 illustrates the response of the synchronously coupled model to the transient emissivity reduction. Displayed is the ensemble mean of 50 realisations for the oceanic mixed layer temperature. As a result of the linear reduction of the atmospheric emissivity, the temperature increase during the first decades is much slower than with instantaneous reduction. Until the final value of the emissivity $\lambda_{A(\text { red })}$ is reached after 100 years, the change rate of the temperature increases with time. Not until two hundred years are simulated, 


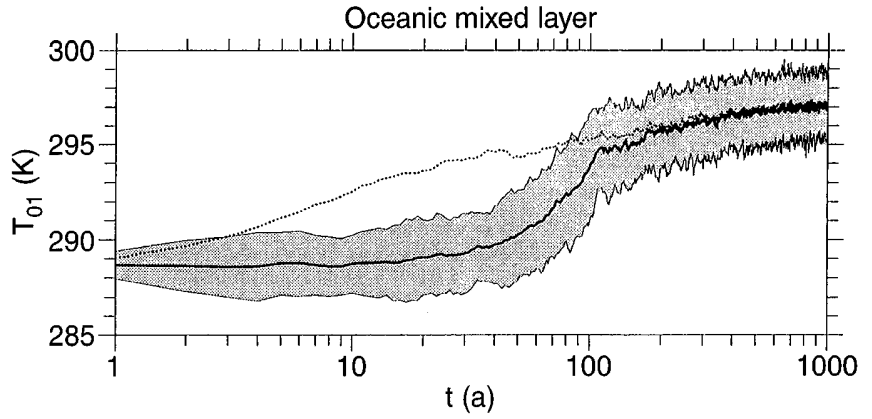

Fig. 9. The annual mean oceanic mixed layer temperature of a synchronously coupled experiment with linear reduction of the atmospheric emissivity. Displayed are the evolution of the ensemble mean of 50 realisations (thick solid curve) and the corresponding curve of the synchronously coupled experiment with instantaneously reduced atmospheric emissivity (dotted curve) (cf. Fig. 2). The shaded areas indicate the range in which $95 \%$ of the annual means occur

is the state of the corresponding experiment with instantaneously reduced emissivity reached.

The periodically synchronously coupled experiments are performed in the same way as the experiments displayed in Fig. 7. As discussed by SV for the model without stochastic forcing, the method with increasing $\tau_{\text {oce }}$ during the initial period is not necessary in the case of the transient emissivity reduction. However, with stochastic forcing, an additional improvement can be achieved, if increasing length of the ocean only periods are used. In the examples displayed in Fig. $10 \tau_{\text {oce }}$ takes the values $6,12,18,24,30,39,48,57$ and 66 months for the rest of the simulations. In con-
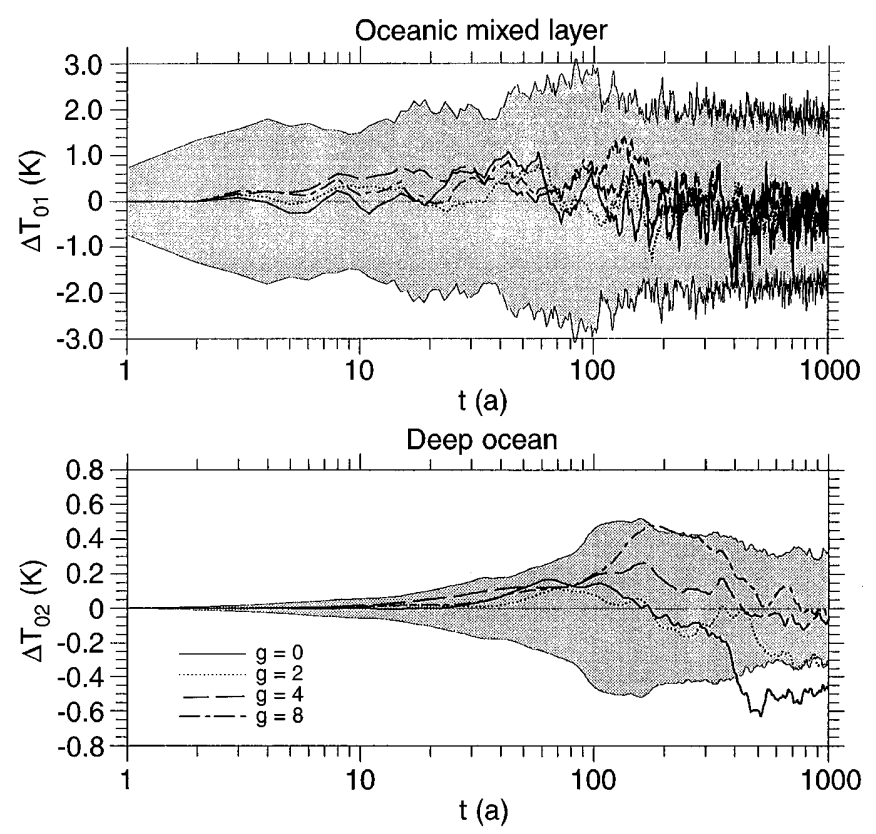

Fig. 10. Errors of the ensemble mean responses to the linear reduction of the atmospheric emissivity as simulated by the periodically synchronously coupled model with different weights $g$. Shaded areas indicate the range in which $95 \%$ of the values of the synchronously coupled reference experiment occur trast with the corresponding experiments with step function forcing no systematic underestimation of the oceanic mixed layer temperature after the first synchronous period occurs. The error of the oceanic mixed layer increases only slightly during the first 200 years. The convergence to a quasi-equilibrium state that is somewhat different from that in the synchronously coupled reference experiment is a common feature of the experiments with step function and linear forcing. The results show that a slower change of the external forcing does not significantly reduce the discrepancies of the deep ocean temperature.

\section{Conclusions}

In order to develop a periodically synchronous coupling technique which is suitable for coupled atmosphere-ocean models with high internal variability, a simple zero-dimensional non-linear energy balance model with stochastic external forcing has been employed. A coupling method has been presented which makes use of weighted annual mean fluxes and of the intra-annual variability of the last 12 months of the newly finished synchronously coupled period. The results show a suitable reproducibility of both the transient response and the equilibrium state as computed by the corresponding synchronously coupled model. The introduction of weighted annual means allows a separation of short-term interannual fluctuations and long-term climate trends.

With respect to the transient behaviour, the best results of the periodically synchronously coupled experiments with step function have been achieved with weights $g$ of 1 or 2 depending on the length of the ocean only periods. For larger weights, a delayed model response has been observed. As the deviations do not leave the range of the fluctuations of the synchronously coupled reference run, the quasi-equilibrium state is reliably reproduced for experiments with weighted fluxes $(g>0)$. Common to all periodically synchronously coupled experiments is the fact that the variability of results from different realisations (i.e. from different initial conditions) is larger than in the synchronously coupled case. This causes a slightly different mean state due to the non-linearity of the model. The magnitude of these various errors strongly depends on the length of the ocean only periods. If the length of these periods is increased both the computational expense and the accuracy of the periodically synchronously coupled simulations decrease.

The main advantage of a periodically synchronous coupling technique is, of course, the enormous reduction of the consumed computer time. In the case of simple models this advantage is not of real importance. However, in the case of more comprehensive models this could be essential. Our tests which have been presented in this paper can only be regarded as a first guess for a more comprehensive model such as a coupled atmosphere-ocean general circulation model. In the simple model, many processes are not considered, 
for example spatially inhomogenities or dynamical processes. In addition, in more comprehensive models the coupling is performed not only with heat fluxes, but also with mass and momentum fluxes which all exhibit different characteristics. In order to test its accuracy, the periodically synchronous coupling technique has to be adapted to a global coupled atmosphereocean general circulation model. The output of the current study can serve as a guideline. A successful test using a comprehensive model will be described in a forthcoming paper.

Acknowledgements. The work was supported by grants from the climatic program of the European Union (EPOC-0003-C(MB) and EV5V-CT92-0123). The authors would like to thank Mr. Kristof Richmond for his advice concerning the English language. We also thank the anonymous reviewers for the helpful comments.

\section{References}

Harvey LDD (1986) Computational efficiency and accuracy of methods for asynchronously coupling atmosphere-ocean models. Part II: testing with a seasonal cycle. J Phys Oceanogr $16: 11-24$
Hasselmann K (1988) Some problems in the numerical simulation of climate variability using high resolution coupled models. In: Schlesinger ME (ed) Physically-based modelling and simulation of climate and climate change - Part I. Kluwer, Dordrecht, pp 583-614

Manabe S, Bryan K (1969) Climate calculations with a combined ocean-atmosphere model. J Atm Sci 26:786-789

Manabe S, Stouffer RJ (1988) Two stable equilibria of a coupled ocean-atmosphere model. J Clim 1:841-866

Roberts DL (1990) An investigation of the Gates asynchronous coupling strategy using a simple energy-balance model. Dyn Atmos Oceans 14:279-301

Sausen R (1988) Asynchronous coupling of ocean and atmosphere models. In: Modelling the sensitivity and variations of the ocean-atmosphere system. Rep Workshop at the ECMWF. WCRP-15, WMO/TD-254:280-289

Sausen R, Voss R (1996) Techniques for asynchronous and periodically-synchronous coupling of atmosphere and ocean models. Part I: general strategy and application to the cyclo-stationary case. Clim Dyn 12:313-323

Schlesinger ME (1979) Discussion of "A global ocean-atmosphere model with seasonal variation for future studies of climate sensitivity". Dyn Atmos Oceans 3:427-432

Schneider SH, Harvey LDD (1986) Computational efficiency and accuracy of methods for asynchronously coupling atmosphere-ocean models. Part I: testing with a mean annual model. J Phys Oceanogr 16:3-10 